\title{
Robust Controller Design for Modified Projective Synchronization of Chen-Lee Chaotic Systems with Nonlinear Inputs
}

\author{
Jui-sheng Lin, ${ }^{1}$ Neng-Sheng Pai, ${ }^{2}$ and Her-Terng Yau ${ }^{2}$ \\ ${ }^{1}$ Department of Electrical Engineering, Far-East University, Tainan 744, Taiwan \\ ${ }^{2}$ Department of Electrical Engineering, National Chin-Yi University of Technology, Taichung 411, Taiwan
}

Correspondence should be addressed to Her-Terng Yau, pan1012@ms52.hinet.net

Received 10 April 2009; Accepted 25 July 2009

Recommended by Mohammad Younis

This study demonstrates the modified projective synchronization in Chen-Lee chaotic system. The variable structure control technology is used to design the synchronization controller with input nonlinearity. Based on Lyapunov stability theory, a nonlinear controller and some generic sufficient conditions can be obtained to guarantee the modified projective synchronization, including synchronization, antisynchronization, and projective synchronization in spite of the input nonlinearity. The numerical simulation results show that the synchronization and antisynchronization can coexist in Chen-Lee chaotic systems. It demonstrates the validity and feasibility of the proposed controller.

Copyright (c) 2009 Jui-sheng Lin et al. This is an open access article distributed under the Creative Commons Attribution License, which permits unrestricted use, distribution, and reproduction in any medium, provided the original work is properly cited.

\section{Introduction}

Chaos is very interesting nonlinear phenomenon and has been intensively studied in the last three decades [1-3]. A fundamental characteristic of a chaotic system is its extreme sensitivity to initial conditions; that is, small differences in the initial state can lead to extraordinary differences in the system state. Since the ideal of synchronizing two identical chaotic systems from different initial conditions was introduced by Carroll and Pecora in 1990, chaos synchronization has received increasing attention, especially in secure communication $[4,5]$. Many methods have been presented for the synchronization of chaotic system [6-11]. However most of these methods are concentrated on studying complete synchronization (CS). In the practical applications, CS only occurs at a certain point in the parameter space, and it is difficult to achieve CS except under ideal conditions. Recently, thus a more general form of synchronization scheme, called generalized synchronization (GS), has been extensively investigated [12-16], where the drive and response system could be synchronized up to a scaling factor $\alpha$. More recently, Li [17] considers a new GS method, called modified projective 
synchronization (MPS), where the responses of the synchronized dynamical states synchronize up to a constant scaling matrix. However, most of research efforts mentioned above have concentrated on the linear control input. Moreover, when the controller is realized in practical physical systems, due to physical limitations of actuators, the nonlinearities in control input do exist. The presence of nonlinearities in control input may cause serious influence of system performance and decrease the system response. Besides, the nonlinearity in control input may cause the chaotic system perturbed to unpredictable results because the chaotic system is very sensitive to any system parameters. Therefore, its effect cannot be ignored in analysis of control design and realization for chaos synchronization. Thus the derivation of controller with input nonlinearity for chaos synchronization is an important problem.

In this paper, the problem of chaos synchronization to Chen-Lee system with input nonlinearity is considered. For MPS of the system, a variable structure control scheme has been proposed. The technique requires two stages. The first stage is to select stable sliding surfaces for the desired dynamics, and the second stage is to design a switching control law to achieve the stable sliding surfaces. Then, the chaos synchronization of the system is proved by the Lypapunov stability theory. Finally, numerical simulation is carried to confirm the validity of the proposed theoretical approach.

\section{Description of the Problem}

Consider two chaotic systems given by

$$
\begin{gathered}
\frac{d x_{m}}{d t}=f\left(x_{m}, t\right), \\
\frac{d x_{s}}{d t}=g\left(x_{s}, t\right)+\phi\left(u\left(x_{m}, x_{s}, t\right)\right),
\end{gathered}
$$

where $x_{m}=\left[\begin{array}{llll}x_{1} & y_{1} & z_{1} & \cdots\end{array}\right]^{T} \in R^{n}, x_{s}=\left[\begin{array}{llll}x_{2} & y_{2} & z_{2} & \cdots\end{array}\right] \in R^{n}, f, g \in C^{r}\left[R^{+} \times\right.$ $\left.R^{n}, R^{n}\right], u \in C^{r}\left[R^{+} \times R^{n} \times R^{n}, R^{n}\right]$, and $r \geq 1, R^{+}$is the set of nonnegative numbers. Assume that (2.1) is the drive system (master system), (2.2) is the response system (slave system), and $\phi\left(u\left(x_{m}, x_{s}, t\right)\right)$ is the nonlinear control input attached in the response system. If for all $x_{m}\left(t_{0}\right), x_{s}\left(t_{0}\right) \in R^{n}, \lim _{t \rightarrow \infty}\left|x_{s_{i}}(t)-\alpha_{i} x_{m_{i}}(t)\right|=0$, for $i=1,2, \ldots, n$, then the response and drive systems are said to be in modified projective synchronization (MPS). In particular, the drive-response systems achieve complete synchronization when all values of $\alpha_{i}$ are equal to 1 . Further, if all values $\alpha_{i}$ are equal to -1 , then two systems are said to be anti-synchronization.

In this paper, our purpose is to achieve the MPS of two identical Chen-Lee chaotic systems by using variable structure control with input nonlinearity. Chen and Lee reported a new chaotic system [18] in 2004, which now called the Chen-Lee system. The system is described by the following nonlinear differential equations and is denoted as the following system:

$$
\begin{aligned}
& \frac{d x}{d t}=-y z+a x, \\
& \frac{d y}{d t}=x z+b y, \\
& \frac{d z}{d t}=\left(\frac{1}{3}\right) x y+c z,
\end{aligned}
$$




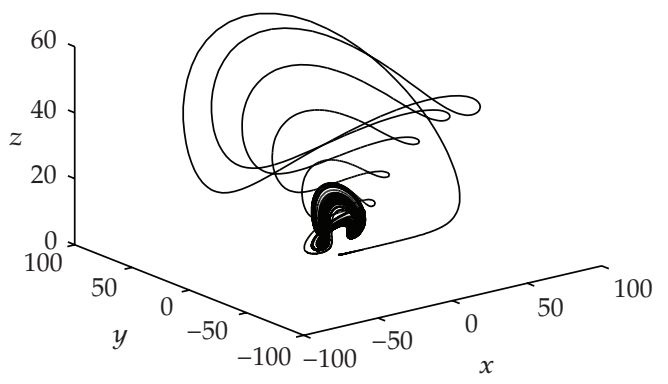

(a)

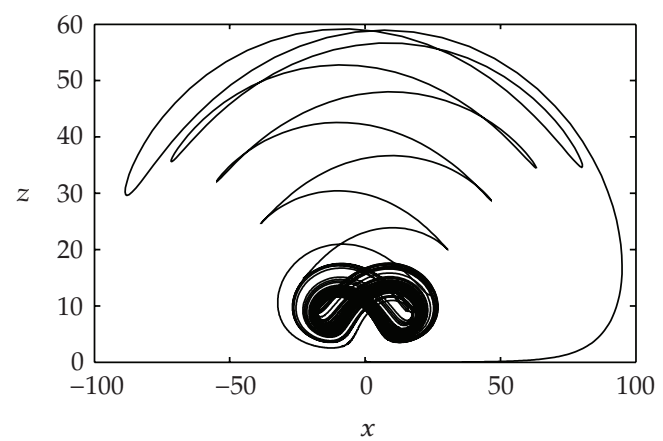

(c)

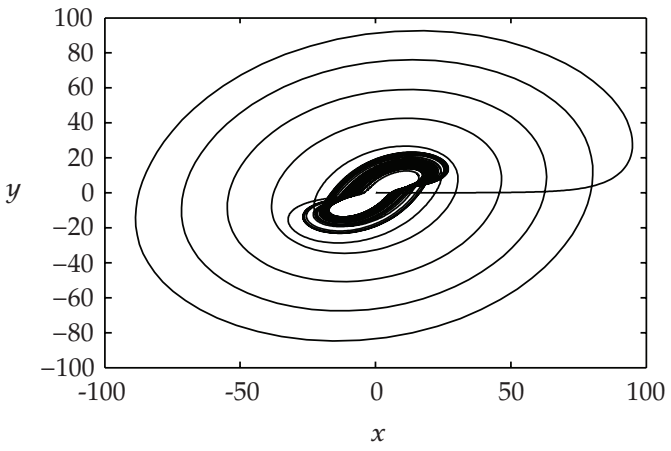

(b)

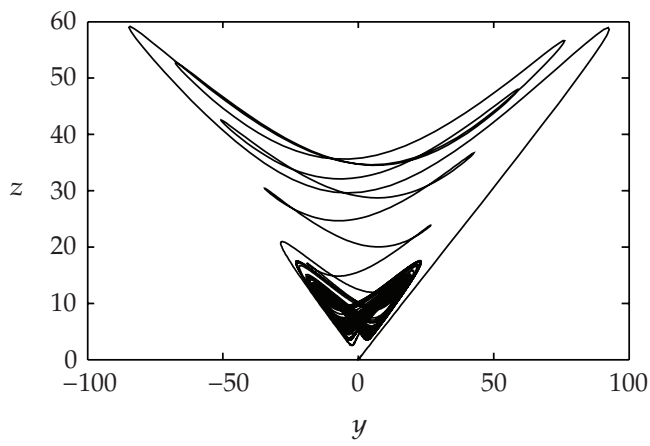

(d)

Figure 1: The phase plane trajectories of Chen-Lee chaotic system with $a=5, b=-10, c=-3.8$.

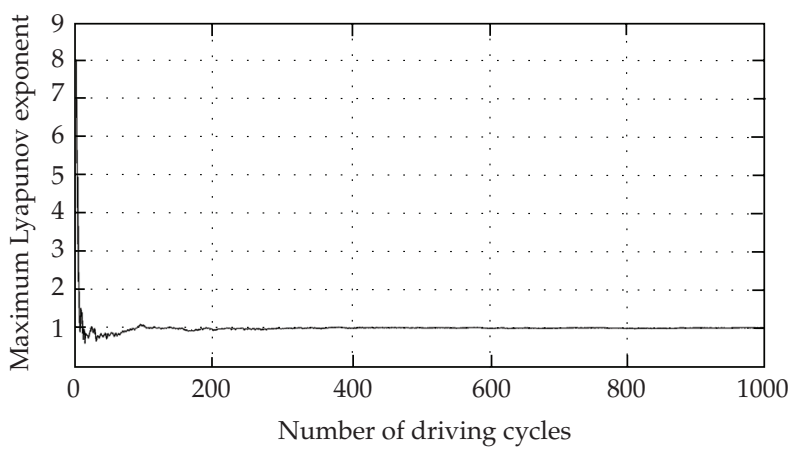

Figure 2: The maximum Lyapunov exponent of Chen-Lee chaotic system plotted as a function of the number of driving cycles with $a=5, b=-10, c=-3.8$.

where $x, y$, and $z$ are state variables, and $a, b$, and $c$ are three system parameters. When $(a, b, c)=(5,-10,-3.8)$, system (2.3) demonstrates a complex attractor as shown in Figure 1. A positive maximum Lyapunov exponent is shown in Figure 2. It shows that the Chen-Lee system is in the state of chaotic motion at this condition. 
For the Chen-Lee chaotic system, the drive and response systems are defined as follows.

Drive system is

$$
\begin{aligned}
& \frac{d x_{1}}{d t}=-y_{1} z_{1}+a x_{1} \\
& \frac{d y_{1}}{d t}=x_{1} z_{1}+b y_{1} \\
& \frac{d z_{1}}{d t}=\left(\frac{1}{3}\right) x_{1} y_{1}+c z_{1} .
\end{aligned}
$$

Response system is

$$
\begin{aligned}
& \frac{d x_{2}}{d t}=-y_{2} z_{2}+a x_{2}+\phi_{1}\left(u_{1}\right) \\
& \frac{d y_{2}}{d t}=x_{2} z_{2}+b y_{2}+\phi_{2}\left(u_{2}\right) \\
& \frac{d z_{2}}{d t}=\left(\frac{1}{3}\right) x_{2} y_{2}+c z_{2}+\phi_{3}\left(u_{3}\right) .
\end{aligned}
$$

where $\phi_{1}\left(u_{1}\right), \phi_{2}\left(u_{2}\right)$, and $\phi_{3}\left(u_{3}\right)$ are the nonlinear control inputs attached in the slave system. Let the synchronization error vector state be $e=\left[\begin{array}{lll}e_{1} & e_{2} & e_{3}\end{array}\right]^{T}=\left[\begin{array}{lll}x_{2}-\alpha_{1} x_{1} & y_{2}-\end{array}\right.$ $\alpha_{2} y_{1} \quad z_{2}-\alpha_{3} z_{1}$ ]. Substitution (2.4) and (2.5) into the error state, the error dynamic equations can be obtained as follows:

$$
\begin{aligned}
& \frac{d e_{1}}{d t}=-e_{2} e_{3}-\alpha_{3} e_{2} z_{1}-\alpha_{2} e_{3} y_{1}+\left(\alpha_{1}-\alpha_{2} \alpha_{3}\right) y_{1} z_{1}+a e_{1}+\phi\left(u_{1}\right), \\
& \frac{d e_{2}}{d t}=e_{1} e_{3}+\alpha_{3} e_{1} z_{1}+\alpha_{1} e_{3} x_{1}+\left(\alpha_{1} \alpha_{3}-\alpha_{2}\right) x_{1} z_{1}+b e_{2}+\phi_{2}\left(u_{2}\right), \\
& \frac{d e_{3}}{d t}=\frac{1}{3}\left(e_{1} e_{2}+\alpha_{2} e_{1} y_{1}+\alpha_{1} e_{2} x_{1}\right)+\frac{1}{3}\left(\alpha_{1} \alpha_{2}-\alpha_{3}\right) x_{1} y_{1}+c e_{3}+\phi_{3}\left(u_{3}\right) .
\end{aligned}
$$

The $\phi_{i}\left(u_{i}(t)\right) \in C^{1}\left(R^{n} \rightarrow R\right)$ is a continues nonlinear function with $\phi_{i}(0)=0$, and $u_{i}(t) \rightarrow$ $\phi_{i}\left(u_{i}(t)\right)$ is inside sector $\left[s_{i}, \rho_{i}\right](i=1,2,3)$, that is,

$$
\varsigma_{i} u_{i}^{2} \leq u_{i} \phi_{i}\left(u_{i}\right) \leq \rho_{i} u_{i}^{2}
$$




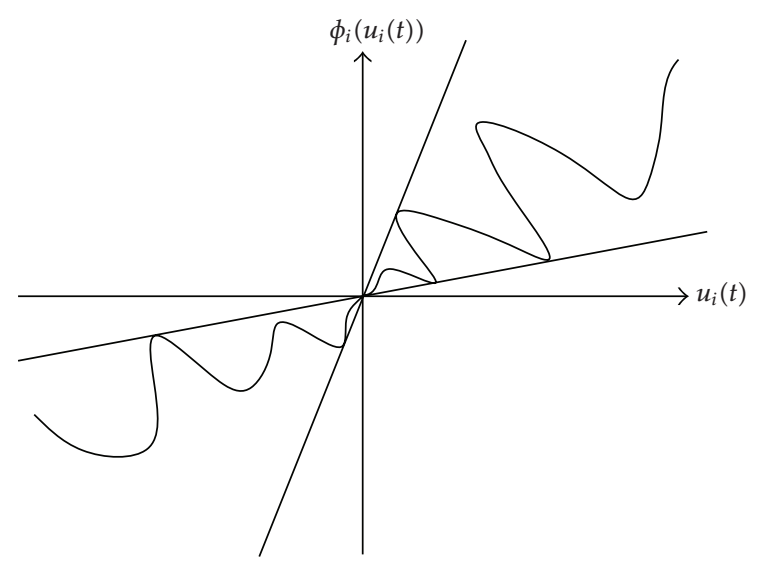

Figure 3: A scalar nonlinear function $\phi_{i}\left(u_{i}(t)\right)$ inside sector $\left[\zeta_{i}, \rho_{i}\right]$.

where $\varsigma_{i}$ and $\rho_{i}$ are nonzero positive constants. A nonlinear function $\phi_{i}\left(u_{i}(t)\right)$ is shown in Figure 3.

Now, the sliding surfaces suitable for the application can be defined as

$$
S_{i}=e_{i}+\int_{0}^{t} \lambda_{i} e_{i}(\tau) d \tau, \quad i=1,2,3
$$

where $S_{i}(t) \in R$ and $\lambda_{i}$ is the design parameters which can be determined later. For the existence of the sliding mode [19], it is necessary and sufficient that

$$
\begin{gathered}
S_{i}=e_{i}+\int_{0}^{t} \lambda_{i} e_{i}(\tau) d \tau=0, \quad i=1,2,3, \\
\frac{d S_{i}}{d t}=\frac{d e_{i}}{d t}+\lambda_{i} e_{i}=0, \quad i=1,2,3 .
\end{gathered}
$$

Therefore, the following sliding mode dynamics can be obtained as

$$
\frac{d e_{i}}{d t}=-\lambda_{i} e_{i}, \quad i=1,2,3
$$

Obviously, if the design parameters $\lambda_{i}>0, i=1,2,3$, the stability of (2.10) is surely guaranteed, that is $\lim _{t \rightarrow \infty} e_{i}(t) \rightarrow 0$. Thus, the response system will be derived to drive system by designing the appropriate signal control inputs $u_{i}(t), i=1,2,3$. 


\section{Description of Variable Structure Control Law with Input Nonlinearity}

We choose a control law of the form

$$
u_{i}=-\gamma_{i} \eta_{i} \operatorname{sign}\left(S_{i}\right), \quad \gamma_{i}>\frac{1}{S_{i}}, i=1,2,3
$$

where

$$
\begin{aligned}
& \eta_{1}=\left|-e_{2} e_{3}-\alpha_{3} e_{2} z_{1}-\alpha_{2} e_{3} y_{1}+\left(\alpha_{1}-\alpha_{2} \alpha_{3}\right) y_{1} z_{1}+a e_{1}+\lambda_{1} e_{1}\right|, \\
& \eta_{2}=\left|e_{1} e_{3}+\alpha_{3} e_{1} z_{1}+\alpha_{1} e_{3} x_{1}+\left(\alpha_{1} \alpha_{3}-\alpha_{2}\right) x_{1} z_{1}+b e_{2}+\lambda_{2} e_{2}\right|, \\
& \eta_{3}=\left|\frac{1}{3}\left(e_{1} e_{2}+\alpha_{2} e_{1} y_{1}+\alpha_{1} e_{2} x_{1}\right)+\frac{1}{3}\left(\alpha_{1} \alpha_{2}-\alpha_{3}\right) x_{1} y_{1}+c e_{3}+\lambda_{3} e_{3}\right| .
\end{aligned}
$$

Based on the control law (3.1), the reaching condition $s(t)(d s(t) / d t)<0$ is guaranteed in the following theorem; that is, the proposed scheme (3.1) will derive the system (2.6) with nonlinear inputs onto the sliding mode $s(t)=0$.

Theorem 3.1. Consider the error dynamics system (2.6) with input nonlinearities. The hitting condition of the sliding mode is satisfied, if the control $u_{i}(t)$ is given by (3.1) for $i=1,2,3,4$.

Proof. Letting the Lyapunov function of the system be $V=(1 / 2)\left(S_{1}{ }^{2}+S_{2}{ }^{2}+S_{3}{ }^{2}\right)$, then its derivative with respect to time is

$$
\begin{aligned}
\frac{d V}{d t}= & S_{1} \frac{d S_{1}}{d t}+S_{2} \frac{d S_{2}}{d t}+S_{3} \frac{d S_{3}}{d t} \\
= & S_{1}\left(\frac{d e_{1}}{d t}+\lambda_{1} e_{1}\right)+S_{2}\left(\frac{d e_{2}}{d t}+\lambda_{2} e_{2}\right)+S_{3}\left(\frac{d e_{3}}{d t}+\lambda_{3} e_{3}\right) \\
= & S_{1}\left(-e_{2} e_{3}-\alpha_{3} e_{2} z_{1}-\alpha_{2} e_{3} y_{1}+\left(\alpha_{1}-\alpha_{2} \alpha_{3}\right) y_{1} z_{1}+a e_{1}+\phi\left(u_{1}\right)+\lambda_{1} e_{1}\right) \\
& +S_{2}\left(e_{1} e_{3}+\alpha_{3} e_{1} z_{1}+\alpha_{1} e_{3} x_{1}+\left(\alpha_{1} \alpha_{3}-\alpha_{2}\right) x_{1} z_{1}+b e_{2}+\phi_{2}\left(u_{2}\right)+\lambda_{2} e_{2}\right) \\
& +S_{3}\left(\frac{1}{3}\left(e_{1} e_{2}+\alpha_{2} e_{1} y_{1}+\alpha_{1} e_{2} x_{1}\right)+\frac{1}{3}\left(\alpha_{1} \alpha_{2}-\alpha_{3}\right) x_{1} y_{1}+c e_{3}+\phi_{3}\left(u_{3}\right)+\lambda_{3} e_{3}\right) \\
\leq & \left|S_{1}\right|\left|-e_{2} e_{3}-\alpha_{3} e_{2} z_{1}-\alpha_{2} e_{3} y_{1}+\left(\alpha_{1}-\alpha_{2} \alpha_{3}\right) y_{1} z_{1}+a e_{1}+\lambda_{1} e_{1}\right|+S_{1} \phi_{1}\left(u_{1}\right) \\
& +\left|S_{2}\right|\left|e_{1} e_{3}+\alpha_{3} e_{1} z_{1}+\alpha_{1} e_{3} x_{1}+\left(\alpha_{1} \alpha_{3}-\alpha_{2}\right) x_{1} z_{1}+b e_{2}+\lambda_{2} e_{2}\right|+S_{2} \phi_{2}\left(u_{2}\right) \\
& +\left|S_{3}\right|\left|\frac{1}{3}\left(e_{1} e_{2}+\alpha_{2} e_{1} y_{1}+\alpha_{1} e_{2} x_{1}\right)+\frac{1}{3}\left(\alpha_{1} \alpha_{2}-\alpha_{3}\right) x_{1} y_{1}+c e_{3}+\lambda_{3} e_{3}\right|+S_{3} \phi_{3}\left(u_{3}\right) \\
\leq & \eta_{1}\left|S_{1}\right|-\varsigma_{1} \gamma_{1} \eta_{1}\left|S_{1}\right|+\eta_{2}\left|S_{2}\right|-\varsigma_{2} \gamma_{2} \eta_{2}\left|S_{2}\right| \eta_{2}\left|S_{2}\right|-\varsigma_{3} \gamma_{3} \eta_{3}\left|S_{3}\right| \\
\leq & \left(1-\gamma_{1} S_{1}\right) \eta_{1}\left|S_{1}\right|+\left(1-\gamma_{2} \varsigma_{2}\right) \eta_{2}\left|S_{2}\right|+\left(1-\gamma_{3} \varsigma_{3}\right) \eta_{3}\left|S_{3}\right|,
\end{aligned}
$$




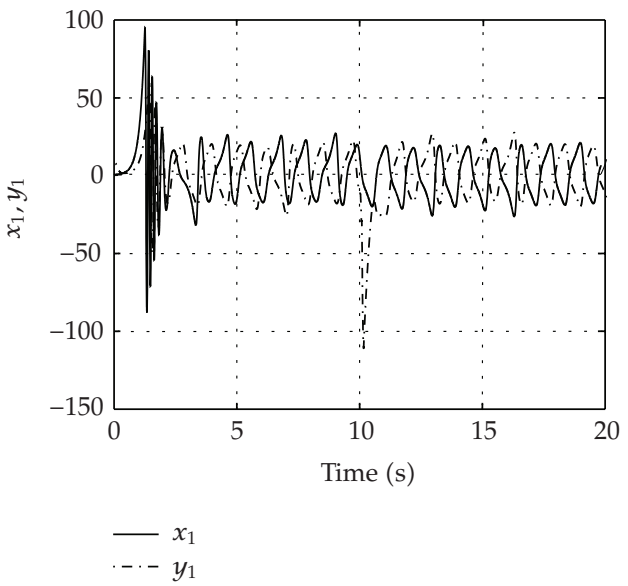

(a)

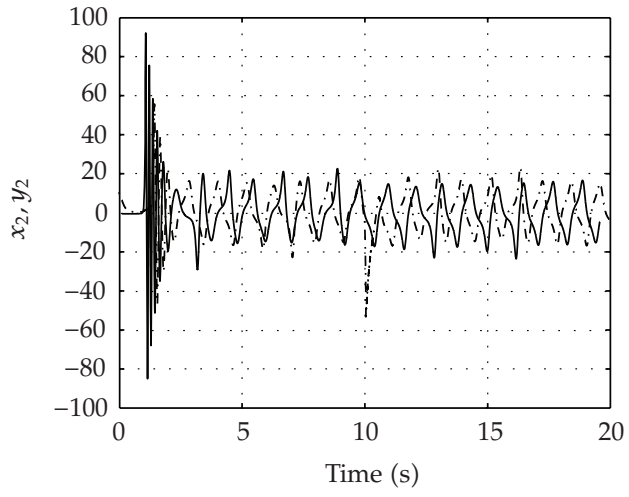

$\begin{aligned} &-x_{2} \\ & \cdots y_{2}\end{aligned}$

(b)

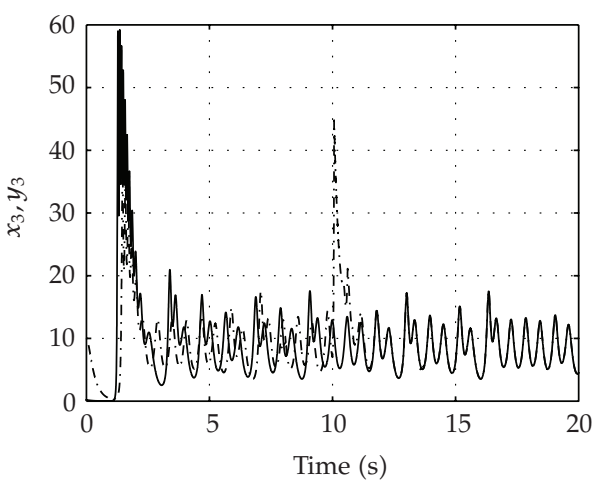

$x_{3}$
$\ldots y_{3}$

(c)

Figure 4: The time history of MPS of controlled drive $\left(x_{1}, x_{2}, x_{3}\right)$ and response $\left(y_{1}, y_{2}, y_{3}\right)$ Chen-Lee chaotic systems: (a) $x_{1}, y_{1}$ versus time $t$; (b) $x_{2}, y_{2}$ versus time $t$; (c) $x_{3}, y_{3}$ versus time $t$. The control is active at $t=10$ seconds.

where

$$
\begin{aligned}
& u_{i} \phi_{i}\left(u_{i}\right) \geq s_{i} u_{i}^{2} \\
& \Longrightarrow-\gamma \eta_{i} \operatorname{sig} u\left(S_{i}\right) \phi\left(u_{i}\right) \geq s_{i} \gamma^{2} \eta_{i}^{2} \operatorname{sign}^{2}\left(S_{i}\right) \\
& \Longrightarrow-\gamma \eta_{i}\left|S_{i}\right| S_{i} \phi\left(u_{i}\right) \geq s_{i} \gamma \eta_{i}\left|S_{i}\right|\left|S_{i}\right|, \quad \text { for } i=1,2,3 . \\
& \Longrightarrow-S_{i} \phi\left(u_{i}\right) \geq s_{i} \gamma_{i} \eta_{i}\left|S_{i}\right| \\
& \Longrightarrow S_{i} \phi\left(u_{i}\right) \leq-\varsigma_{i} \gamma_{i} \eta_{i}\left|S_{i}\right| .
\end{aligned}
$$

Therefore, if

$$
\gamma_{i}>\frac{1}{\zeta_{i}}, \quad \text { for } i=1,2,3
$$




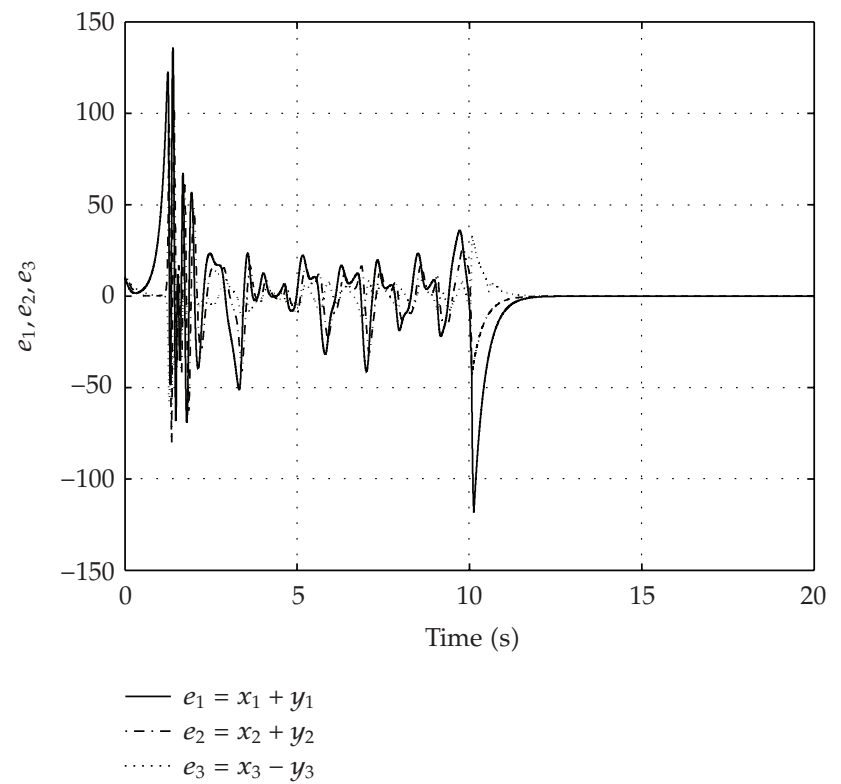

Figure 5: Synchronization errors $e_{1}, e_{2}, e_{3}$ versus time $t$. The control is active at $t=10$ seconds.

then $d V / d t<0$, confirming the presence of reaching condition. Thus the proof is achieved completely.

\section{Numerical Studies}

In this simulation, the 4th-order Runge-Kutta algorithm was used to solve the sets of differential equations related to the drive and response systems with a time grid of 0.0001 . The initial values of drive and response Chen-Lee chaotic system are $\left[\begin{array}{lll}x_{1}(0) & y_{1}(0) & z_{1}(0)\end{array}\right]=$ $\left[\begin{array}{lll}0.2 & 0.2 & 0.2\end{array}\right],\left[\begin{array}{lll}x_{2}(0) & y_{2}(0) & z_{2}(0)\end{array}\right]=\left[\begin{array}{lll}10 & 10 & 10\end{array}\right]$. In the synchronization example, we selected $\lambda_{1}=\lambda_{2}=\lambda_{3}=3$ to result in stable sliding modes and the nonlinear inputs are defined as

$$
\phi_{i}\left(u_{i}(t)\right)=\left[0.7+0.2 \cdot \sin \left(u_{i}(t)\right)\right] u_{i}(t), \quad i=1,2,3 .
$$

Furthermore, it is assumed that the slope of nonlinear sectors in these three synchronization examples is $\varsigma_{1}=\varsigma_{2}=\varsigma_{3}=0.5$ and $\rho_{1}=\rho_{2}=\rho_{3}=0.9$, and the parameters $\gamma_{1}=\gamma_{2}=$ $\gamma_{3}=5$ are selected to satisfy the condition (3.1). The time responses of controlled driveresponse Chen-Lee systems are shown in Figures 4(a)-4(c). It can be see that the response system synchronizes with the drive system in spite of input nonlinearity. Obviously, the synchronization errors converge asymptotically to zero after the control is active at time $t=10$ seconds in Figure 5 . 


\section{Conclusions}

In this paper, we investigate the hybrid projective synchronization of controlled Chen-Lee chaotic system with input nonlinearity. Based on Lyapunov stability theorem, an effective control method for synchronizing two identical Chen-Lee chaotic systems with different initial conditions has been proposed using variable structure design. The proposed nonlinear control enables stabilization of synchronization error dynamics to zeros asymptotically in spite of input nonlinearity. Numerical simulation results are presented to show that the synchronization and antisynchronization coexist by the proposed synchronization technique. The main feature of this approach is that it gives the flexibility to construct a control law so that the control strategy can be easily extended to any other types of chaotic systems.

\section{Acknowledgment}

The financial support of this research by the National Science Council of Taiwan, under Grant no. NSC96-2221-E-269-010-MY2 is greatly appreciated.

\section{References}

[1] L. M. Pecora and T. L. Carroll, "Synchronization in chaotic systems," Physical Review Letters, vol. 64, no. 8, pp. 821-824, 1990.

[2] T. L. Carroll and L. M. Pecora, "Synchronizing chaotic circuits," IEEE Transactions on Circuits and Systems, vol. 38, no. 4, pp. 453-456, 1991.

[3] G. Chen and X. Dong, From Chaos to Order: Methodologies, Perspectives and Applications, vol. 24 of World Scientific Series on Nonlinear Science. Series A: Monographs and Treatises, World Scientific, River Edge, NJ, USA, 1998.

[4] L. Kocarev and U. Parlitz, "General approach for chaotic synchronization with applications to communication," Physical Review Letters, vol. 74, no. 25, pp. 5028-5031, 1995.

[5] K. Murali and M. Lakshmanan, "Secure communication using a compound signal from generalized synchronizable chaotic systems," Physics Letters A, vol. 241, no. 6, pp. 303-310, 1998.

[6] H. Salarieh and A. Alasty, "Adaptive synchronization of two chaotic systems with stochastic unknown parameters," Communications in Nonlinear Science and Numerical Simulation, vol. 14, no. 2, pp. 508-519, 2009.

[7] G. S. M. Ngueuteu, R. Yamapi, and P. Woafo, "Effects of higher nonlinearity on the dynamics and synchronization of two coupled electromechanical devices," Communications in Nonlinear Science and Numerical Simulation, vol. 13, no. 7, pp. 1213-1240, 2008.

[8] S. Bowong, "Adaptive synchronization between two different chaotic dynamical systems," Communications in Nonlinear Science and Numerical Simulation, vol. 12, no. 6, pp. 976-985, 2007.

[9] H.-T. Yau, J.-S. Lin, and J.-J. Yan, "Synchronization control for a class of chaotic systems with uncertainties," International Journal of Bifurcation and Chaos, vol. 15, no. 7, pp. 2235-2246, 2005.

[10] H.-T. Yau, "Design of adaptive sliding mode controller for chaos synchronization with uncertainties," Chaos, Solitons \& Fractals, vol. 22, no. 2, pp. 341-347, 2004.

[11] H.-T. Yau, C.-L. Kuo, and J.-J. Yan, "Fuzzy sliding mode control for a class of chaos synchronization with uncertainties," International Journal of Nonlinear Sciences and Numerical Simulation, vol. 7, no. 3, pp. 333-338, 2006.

[12] N. F. Rulkov, M. M. Sushchik, L. S. Tsimring, and H. D. I. Abarbanel, “Generalized synchronization of chaos in directionally coupled chaotic systems," Physical Review E, vol. 51, no. 2, pp. 980-994, 1995

[13] Y.-W. Wang and Z.-H. Guan, "Generalized synchronization of continuous chaotic system," Chaos, Solitons \& Fractals, vol. 27, no. 1, pp. 97-101, 2006.

[14] G.-L. Wen and D. Xu, “Observer-based control for full-state projective synchronization of a general class of chaotic maps in any dimension," Physics Letters A, vol. 333, no. 5-6, pp. 420-425, 2004.

[15] G. Wen and D. Xu, "Nonlinear observer control for full-state projective synchronization in chaotic continuous-time systems," Chaos, Solitons \& Fractals, vol. 26, no. 1, pp. 71-77, 2005. 
[16] G.-H. Li, "Generalized projective synchronization between Lorenz system and Chen's system," Chaos, Solitons \& Fractals, vol. 32, no. 4, pp. 1454-1458, 2007.

[17] G.-H. Li, "Modified projective synchronization of chaotic system," Chaos, Solitons E Fractals, vol. 32, no. 5, pp. 1786-1790, 2007.

[18] H.-K. Chen and C.-I. Lee, "Anti-control of chaos in rigid body motion," Chaos, Solitons \& Fractals, vol. 21, no. 4, pp. 957-965, 2004.

[19] J. E. Slotine and W. Li, Applied Nonlinear Control, Prentice-Hall, Englewood Cliffs, NJ, USA, 1991. 


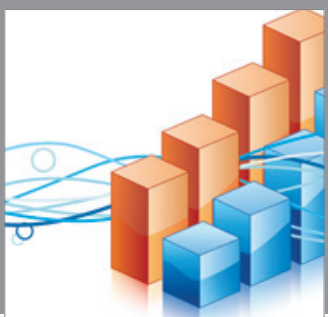

Advances in

Operations Research

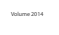

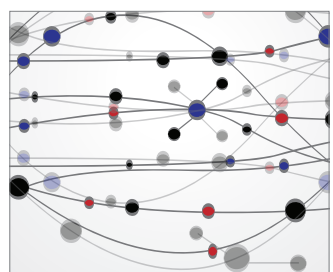

\section{The Scientific} World Journal
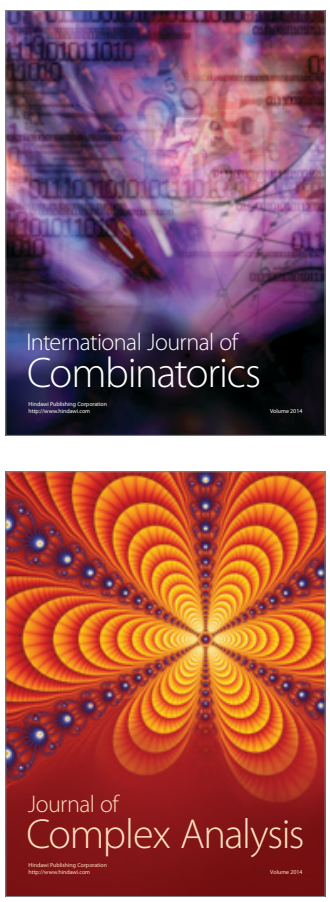

International Journal of

Mathematics and

Mathematical

Sciences
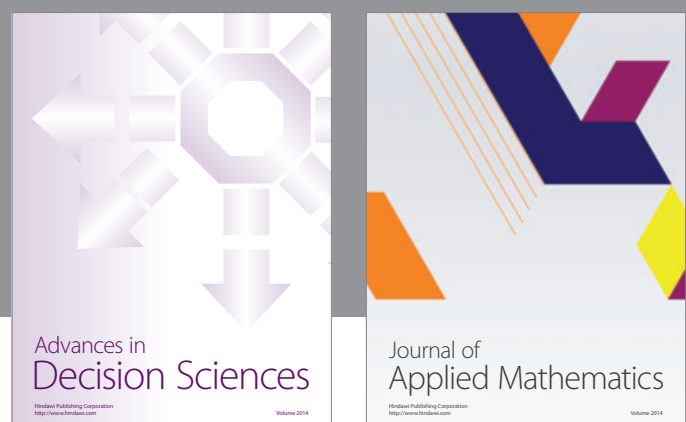

Journal of

Applied Mathematics
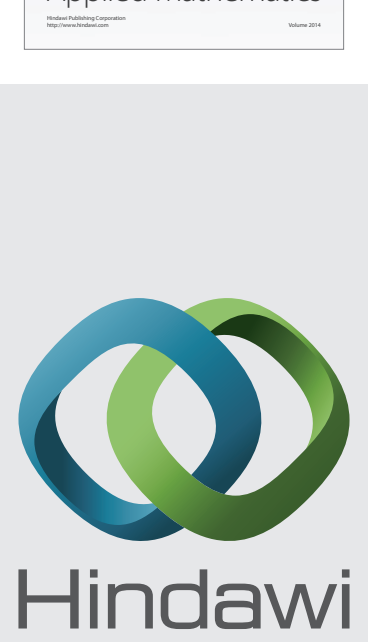

Submit your manuscripts at http://www.hindawi.com
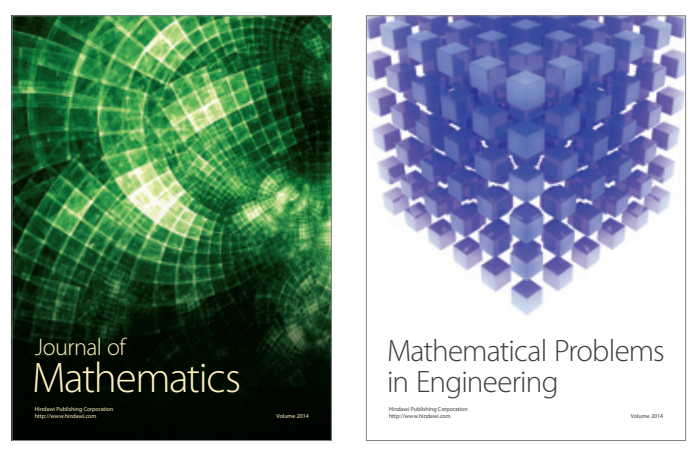

Mathematical Problems in Engineering
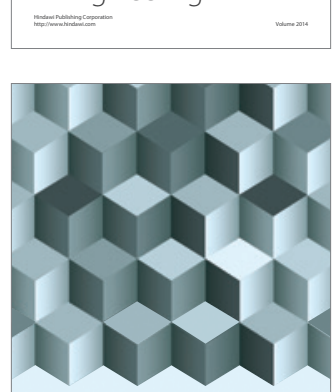

Journal of

Function Spaces
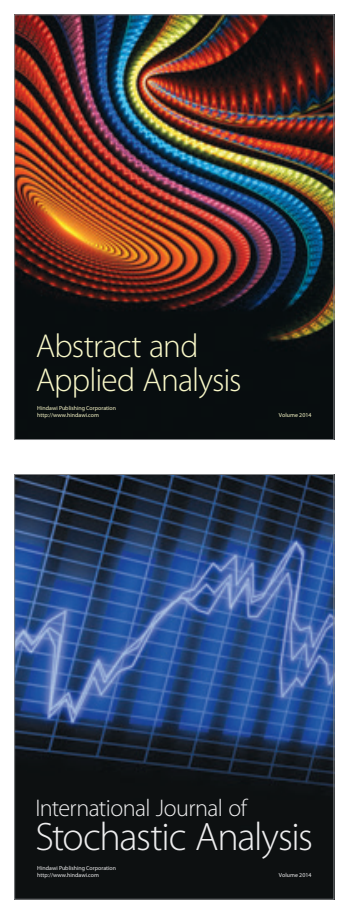

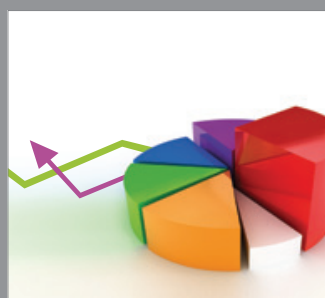

ournal of

Probability and Statistics

Promensencen
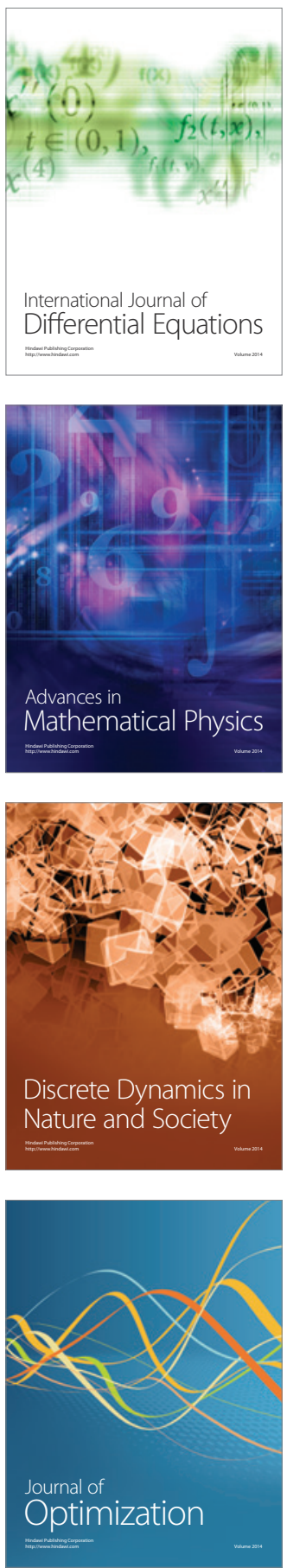